MITSUBISHI ELECTRIC RESEARCH LABORATORIES

http://www.merl.com

\title{
A Stochastic Geometry Analysis of Cooperative Wireless Networks Powered by Energy Harvesting
}

\author{
Khan, T.; Orlik, P.V.; Kim, K.J. \\ TR2015-078 June 2015
}

\begin{abstract}
A large wireless network with energy harvesting transmitters is considered, where a group of $\mathrm{K}$ transmitters forma cluster to cooperatively serve a desired user. Using stochastic geometry, simple closed-form expressions are derived to characterize the outage performance as a function of important parameters such as the energy harvesting rate, buffer size and cluster size for a given cluster geometry. The developed framework also allows the $\mathrm{K}$ in-cluster transmitters to have different energy harvesting capabilities. A comparison with simulation results reveals that the derived expressions closely model the signal-to-interference and-noise ratio distribution at the receiver, particularly in the low-outage regime. Lastly, the developed framework is used to investigate the impact of different parameters such as cluster and buffer size on outage performance.
\end{abstract}

2015 IEEE International Conference on Communications (ICC)

\footnotetext{
This work may not be copied or reproduced in whole or in part for any commercial purpose. Permission to copy in whole or in part without payment of fee is granted for nonprofit educational and research purposes provided that all such whole or partial copies include the following: a notice that such copying is by permission of Mitsubishi Electric Research Laboratories, Inc.; an acknowledgment of the authors and individual contributions to the work; and all applicable portions of the copyright notice. Copying, reproduction, or republishing for any other purpose shall require a license with payment of fee to Mitsubishi Electric Research Laboratories, Inc. All rights reserved.
} 



\title{
A Stochastic Geometry Analysis of Cooperative Wireless Networks Powered by Energy Harvesting
}

\author{
Talha Ahmed Khan*, Philip Orlik ${ }^{\dagger}$ and Kyeong Jin Kim ${ }^{\dagger}$ \\ *Dept. of Electrical and Computer Engineering, The University of Texas at Austin, TX 78712, USA \\ ${ }^{\dagger}$ Mitsubishi Electric Research Laboratories (MERL), Cambridge, MA 02139, USA \\ Email: talhakhan@utexas.edu, \{porlik, kkim\}@merl.com
}

\begin{abstract}
A large wireless network with energy harvesting transmitters is considered, where a group of $K$ transmitters form a cluster to cooperatively serve a desired user. Using stochastic geometry, simple closed-form expressions are derived to characterize the outage performance as a function of important parameters such as the energy harvesting rate, buffer size and cluster size for a given cluster geometry. The developed framework also allows the $K$ in-cluster transmitters to have different energy harvesting capabilities. A comparison with simulation results reveals that the derived expressions closely model the signal-to-interferenceand-noise ratio distribution at the receiver, particularly in the low-outage regime. Lastly, the developed framework is used to investigate the impact of different parameters such as cluster and buffer size on outage performance.
\end{abstract}

Index Terms-Energy harvesting, stochastic geometry, cooperative wireless networks.

\section{INTRODUCTION}

Recent trends in networking suggest that wireless networks are expected to get more dense and heterogeneous in future [1]. Due to exploding traffic demands and aggressive frequency re-use, wireless link performance is generally believed to be interference-limited. However, if the interfering transmitters are allowed to cooperate, these interfering signals could instead be converted to useful ones. Motivated by this information-theoretic insight [2], there has been tremendous interest in designing cooperative transmission policies for wireless networks (see [3]-[5] and references therein). Such a cooperative setup generally consists of a group of transmitters (henceforth referred to as a cluster) that cooperate by jointly encoding the transmit symbol and/or sharing data/control information (over backhaul links), while the rest of the transmitters act as sources of interference. In this paradigm, the gains from cooperation have been shown to scale with the cluster size [5]. However, with the increase in cluster size, the channel estimation/control overheads and the backhaul requirements also grow, which essentially limit the gains from cooperation [2].

In pursuit of a green and sustainable wireless network, and given the advancement in solid state technologies, wireless devices of the future are expected to be powered by the environment [6]. To this end, there has been considerable research on the front of energy harvesting [7], [8]. With random energy arrivals and a finite energy storage capacity, the

This work was done while T. A. Khan was an intern at MERL. energy availability at the transmitters is rather unpredictable and depends on the energy harvesting technology. This calls for a rethinking of conventional communication protocols. In this regard, several papers have proposed optimal transmission policies assuming causal or non-causal knowledge about energy arrivals for different setups. For example, a point-point link [8], [9], an interference channel [10], and a broadcast channel [11] have been considered. While prior research has mostly investigated isolated setups, some recent studies deal with the network-level dynamics in large noncooperative wireless networks powered by energy harvesting. In [12] and [13], spatial throughput was derived for a selfpowered adhoc network by leveraging tools from stochastic geometry. Similarly, stochastic geometry has also been used for modeling cognitive networks with opportunistic energy harvesting [14], as well as self-powered heterogeneous cellular networks [15]. By modeling wireless networks in a stochastic geometry framework, tractable analytical expression can be developed to get general performance insights, thus obviating the need of exhaustive simulations [16].

In this work, a large self-powered wireless network is considered where all the transmitters access the medium following a random-access protocol. To reap the benefits of cooperation, they are grouped into clusters such that all the in-cluster transmitters jointly serve a common receiver, which is subjected to interference from out-of-cluster nodes. To the best of our knowledge, the performance of such a cooperative self-powered wireless network in a stochastic geometry framework, has not been analyzed, and is therefore the topic of this work. Using stochastic geometry, we derive simple closed-form expressions that characterize the system performance as a function of system parameters (e.g., energy harvesting rate) and cluster geometry, amid interference and noise. Unlike previous work, the developed framework also allows the in-cluster transmitters to have different energy harvesting capabilities. Simulation results indicate that the derived expressions closely model the instantaneous signal-tointerference-and-noise ratio (SINR) distribution at the receiver. In addition, we also investigate the impact of energy harvesting rate and buffer size on outage performance. We now describe the system model in section II. The analytical expressions are derived in section III. Section IV includes the simulation results and the paper is concluded in section V. 


\section{SySTEM Model}

We now describe the system model in detail beginning with the energy harvesting model.

\section{A. Energy Harvesting Model}

We consider a large wireless network consisting of transmitters or access points (APs) which harvest energy using unconventional (e.g., solar) sources. None of the transmitters are privy to non-causal information about energy arrivals, which is assumed to be random and independent across nodes. Without loss of generality, we now describe the energy harvesting model for a typical transmitter equipped with an energy buffer of size $S \in \mathbb{N}$. The energy arrives at the buffer with rate $\rho$ following an independent and identically distributed (i.i.d.) Bernoulli process, i.e., with probability $\rho$, one unit of energy arrives at the buffer in time-slot $t$, while $1-\rho$ is the probability that no energy arrives at the buffer in that slot. A node may choose to transmit with fixed power $P$ if it has sufficient energy in the buffer. No power control is assumed, therefore each transmission depletes the buffer of $P$ units of energy. The energy arrivals are modeled using a birthdeath Markov process (cf. Fig. 1) along the lines of [12], [13].

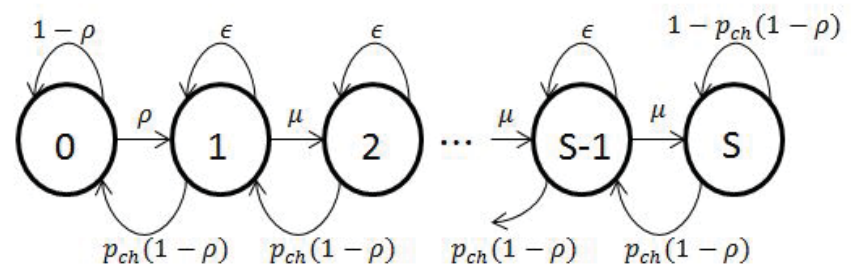

Fig. 1. Finite-state birth-death Markov chain for an energy buffer of size $S$ with $\mu=\rho\left(1-p_{\mathrm{ch}}\right)$ and $\epsilon=\rho p_{\mathrm{ch}}+(1-\rho)\left(1-p_{\mathrm{ch}}\right)$.

For medium access, we consider a fully-distributed random access protocol (slotted ALOHA) where in each time-slot, a node (having sufficient energy) accesses the medium with probability $p_{\mathrm{ch}}$ independently of other nodes. Let $p_{S}=$ $\operatorname{Pr}\left\{A_{S}(t) \geq P\right\}$, where $A_{S}(t)$ denotes the state (i.e., energy level) of the buffer at time $t$. Here, $p_{S}$ denotes the probability that a node has requisite amount of energy available in the buffer of size $S$. Without loss of generality, we assume $P=1$ for ease of exposition in the rest of the sequel. We now define $p_{\text {tr }}$, the transmission probability of an arbitrary node, and express it as a function of system parameters.

Lemma 1: For energy arrivals with rate $\rho>0$, finite energy buffer of size $S \in \mathbb{N}$, and channel access probability $p_{\text {ch }}>0$, we have $p_{\text {tr }}=p_{\text {ch }} p_{S}$ in steady state, where

$$
p_{S}= \begin{cases}\frac{\rho}{\rho+p_{\mathrm{ch}}-\rho p_{\mathrm{ch}}} & S=1 \\ \frac{\rho}{p_{\mathrm{ch}}\left(1-\left(\frac{\rho\left(1-p_{\mathrm{ch}}\right)}{p_{\mathrm{ch}}(1-\rho)}\right)^{S}\right)} & S>1, \rho \neq p_{\mathrm{ch}} \\ \frac{S-\frac{\rho}{p_{\mathrm{ch}}}\left(\frac{\rho\left(1-p_{\mathrm{ch}}\right)}{p_{\mathrm{ch}}(1-\rho)}\right)^{S}}{S+1-\rho} & S>1, \rho=p_{\mathrm{ch}} .\end{cases}
$$

Proof: It can be proved by solving the balance equations for the Markov chain shown in Fig. 1 (e.g., see [12]).

Note that $0<p_{\text {tr }} \leq p_{\text {ch }}$ since $\lim _{\rho \rightarrow 1} p_{\text {tr }}=p_{\text {ch }}$, where $\rho=1$ corresponds to the case when the node is powered by conventional power sources. Furthermore, we consider $p_{\text {ch }}$ to be fixed throughout the network. Therefore, the transmission probability of a node varies as a function of the energy harvesting rate and buffer size. In other words, the higher the $p_{\text {tr }}$ of an AP, the more superior the energy harvesting capability (i.e., harvesting rate and/or buffer size).

\section{B. Network Model}

In our setup, a cluster of $K$ cooperating APs jointly serve a desired user over the same time-frequency resource block. It is assumed that each user is served by the $K$ closest APs. The AP locations are drawn from a homogeneous PPP of intensity $\lambda$, which we denote as $\phi(\lambda)$. Similarly, the user locations are modeled using another PPP $\phi_{u}\left(\lambda_{u}\right)$, which is assumed to be independent of $\phi(\lambda)$. We further assume that the AP density is sufficiently high relative to the user density such that with a high probability, no two users share the same set of closest APs. Leveraging Slivnyak's theorem [16], we consider a typical user located at origin, and characterize the performance in the presence of co-channel interference and noise.

Let $\left\{d_{i}\right\}_{i=1}^{\infty}$ model the Euclidean distance between the typical user and the points belonging to a realization of $\phi$. For each in-cluster AP, we also define normalized distances $\left\{\omega_{i}\right\}_{i=1}^{K}$, where $\omega_{i}=d_{i} / d_{K}$. We now describe the channel model in detail. Let $h_{i}$ be the small-scale fading coefficient for the wireless link between node $i$ and the receiver. It is assumed that all the links experience i.i.d. block-flat Rayleigh fading such that $h_{i} \sim \mathcal{N}_{c}(0,1)$, while the corresponding channel power gain $H_{i}=\left|h_{i}\right|^{2}$ is exponential with unit mean (i.e., $H_{i} \sim \exp (1)$ ). Assuming perfect channel knowledge, each in-cluster AP $i$ uses linear beamforming with coefficient $v_{i}=h_{i}^{*} /\left|h_{i}\right|$ to transmit the same information symbol $s$ where $\mathrm{E}\left[|s|^{2}\right]=1$ (along the lines of [17]). Note that this transmission scheme does not require joint encoding at the transmitters and thus avoids excessive information flow among the in-cluster APs. The received signal at the user can be expressed as

$$
y(t)=\sum_{i=1}^{K} \sqrt{\mathbb{1}_{i}(t) d_{i}^{-\eta}} h_{i}(t) v_{i}(t) s(t)+i(t)+n(t)
$$

where the indicator function $\mathbb{1}_{i}(t)$ models the uncertainty in transmission such that $\operatorname{Pr}\left\{\mathbb{1}_{i}(t)=1\right\}=p_{\mathrm{tr}, i}$ and $\operatorname{Pr}\left\{\mathbb{1}_{i}(t)=\right.$ $0\}=1-p_{\mathrm{tr}, i} \equiv q_{\mathrm{tr}, i}$. The receiver noise $n(t)$ is assumed to be i.i.d. zero-mean complex Gaussian with variance $\sigma^{2}$. Here, the interference term $i(t)=\sum_{i=K+1}^{\infty} \sqrt{\mathbb{1}_{i}(t) d_{i}^{-\eta}} h_{i}(t) \hat{v}_{i}(t) s_{i}(t)$ consists of the signals transmitted by the out-of-cluster APs. Note that $\hat{v}_{i}$ is independent of $h_{i}$ as it is matched to the intended receiver of the interfering APs. It can be seen that $\mathrm{E}\left|h_{i} v_{i}\right|^{2}=1$ for the in-cluster links while $\mathrm{E}\left|h_{i} \hat{v}_{i}\right|^{2}=1$ also for the interfering links. Assuming that interference is 
treated as noise for decoding, a successful packet reception at the receiver can be characterized using the instantaneous SINR which is expressed below (dropping the time index for simplicity)

$$
\operatorname{SINR}_{u}=\frac{\left|\sum_{i=1}^{K} \sqrt{\mathbb{1}_{i} d_{i}^{-\eta}}\right| h_{i}|s|^{2}}{\mathrm{I}+\sigma^{2}}>\frac{\sum_{i=1}^{K} \mathbb{1}_{i} d_{i}^{-\eta} H_{i}}{\mathrm{I}+\sigma^{2}} \equiv \gamma
$$

where $\mathrm{I}=\sum_{i=K+1}^{\infty} \mathbb{1}_{i} d_{i}^{-\eta} H_{i}$ denotes the instantaneous aggregate interference power. In the sequel, we will use $\gamma$, a lower bound on the instantaneous received SINR, for performance analysis. Later, numerical results show that this bound is tight for most settings of interest.

Notation: For ease of exposition, we recall that $q_{\mathrm{tr}} \equiv 1-p_{\mathrm{tr}}$ and define $\Xi=\left\{q_{\mathrm{tr}, i}, \cdots, q_{\mathrm{tr}, K}\right\} \cup\left\{q_{\mathrm{tr}, o}\right\}$, where $\left\{p_{\mathrm{tr}, i}\right\}_{i=1}^{K}$ model the in-cluster transmission probabilities while $p_{\text {tr }, o}$ gives the transmission probability of out-of-cluster APs. We further define $Q=\prod_{i=1}^{K} q_{\mathrm{tr}, i}, \Omega=\left\{\omega_{1}^{\eta}, \cdots, \omega_{K}^{\eta}\right\}, \hat{\Omega}=$ $\left\{\frac{\omega_{1}^{\eta}}{q_{\mathrm{r}, 1}}, \cdots, \frac{\omega_{K}^{\eta}}{q_{\mathrm{r}, K}}\right\}$ and

$$
\alpha_{i}(\Omega)=(-1)^{i} \sum^{+}\left[\left(\begin{array}{c}
K \\
K-i
\end{array}\right)_{\Omega}\right]
$$

where $\sum^{+}[\cdot]$ gives the sum of the elements of the set that it operates on. With a slight abuse of notation, $\left(\begin{array}{c}K \\ K-i\end{array}\right)$ is defined to be the set of all products of the elements of $\Omega$ taken $K-i$ at a time. The summation in (4) is taken over the elements of the set $\left(\begin{array}{c}K \\ K-i\end{array}\right)$. Similarly, the definition of $\alpha_{i}(\hat{\Omega})$ follows from (4) with the set $\Omega$ now replaced by $\hat{\Omega}$.

\section{Stochastic GeOMetry Analysis}

In this section, we derive closed-form expressions for the complementary cumulative distribution function (CCDF) of $\gamma$ as a function of network parameters and cluster geometry.

Theorem 1: For a cluster of size $K$, the CCDF of $\gamma$, $\bar{F}_{\gamma}(K, \theta)=\operatorname{Pr}\{\gamma>\theta\}$, can be expressed in terms of the interference intensity $(\lambda)$, noise power $\left(\sigma^{2}\right)$, energy harvesting parameters $(\Xi)$ and cluster geometry $\left(\left\{d_{i}\right\}_{i=1}^{K}\right)$ as

$$
\bar{F}_{\gamma}(K, \theta)=\left(\frac{\sum_{i=0}^{K-1}\left(\alpha_{i}(\hat{\Omega})-\alpha_{i}(\Omega)\right)\left(\omega_{j}^{\eta}\right)^{i}}{\omega_{j}^{\eta}\left(\prod_{l \neq j}^{K} \omega_{l}^{\eta}-\omega_{j}^{\eta}\right)}\right) \Delta_{j}(\theta)
$$

where

$$
\Delta_{j}(\theta)=e^{-d_{j}^{\eta} \theta \sigma^{2}} e^{-\pi p_{\mathrm{t}, o} \lambda d_{K}^{2} \mathcal{F}\left(\omega_{j}^{\eta} \theta, \eta\right)}
$$

and

$$
\mathcal{F}(U, V)=\frac{2 U}{V-2}{ }_{2} F_{1}\left(1,1-\frac{2}{V}, 2-\frac{2}{V},-U\right)
$$

where ${ }_{2} F_{1}(\cdot)$ is the Gauss hypergeometric function.
Proof: Using (3), we write $\bar{F}_{\gamma}(K, \theta)=\operatorname{Pr}\{\gamma>\theta\}=$ $\mathrm{E}\left[\operatorname{Pr}\left\{S_{K}>\theta d_{K}^{\eta}\left(\mathrm{I}+\sigma^{2}\right)\right\}\right]$ where $S_{K}=\sum_{i=1}^{K} \mathbb{1}_{i} \hat{H}_{i}$ and $\hat{H}_{i}=H_{i} \omega_{i}^{-\eta}$. To proceed further, we first find the CCDF of $S_{K}$, where $S_{K}$ is a sum of $K$ independent random variables. Note that $\hat{H}_{i}$ is exponentially distributed with mean $\omega_{i}^{-\eta}$, whereas the indicator function follows a Bernoulli distribution with mean $p_{\mathrm{tr}, i}$, independently of $\hat{H}_{i}$. The CCDF of $S_{K}$ can be expressed as $(x \geq 0)$

$$
Q \bar{F}_{S_{k}}(x)=\left(\frac{\sum_{i=0}^{K-1}\left(\alpha_{i}(\hat{\Omega})-\alpha_{i}(\Omega)\right)\left(\omega_{j}^{\eta}\right)^{i}}{\omega_{j}^{\eta}\left(\prod_{l \neq j}^{K} \omega_{l}^{\eta}-\omega_{j}^{\eta}\right)}\right) e^{-\omega_{j}^{\eta} x}
$$

where the above expression can be obtained by finding the characteristic function of $S_{K}$, applying partial fraction expansion and then taking the inverse transform, similar to the approach in [18]. Conditioning on the aggregate interference power I, we can write $\bar{F}_{\gamma \mid \mathrm{I}}(K, \theta)=\bar{F}_{S_{K}}\left(K, \theta d_{K}^{\eta}\left(\mathrm{I}+\sigma^{2}\right)\right)$. Using (8), and unconditioning w.r.t. I, we can express $\bar{F}_{\gamma}(K, \theta)$ (for $\theta \geq 0$ ) as

$$
\begin{aligned}
& \bar{F}_{\gamma}(K, \theta)= \\
& Q \sum_{j=1}^{K}\left(\frac{\sum_{i=0}^{K-1}\left(\alpha_{i}(\hat{\Omega})-\alpha_{i}(\Omega)\right)\left(\omega_{j}^{\eta}\right)^{i}}{\omega_{j}^{\eta}\left(\prod_{l \neq j}^{K} \omega_{l}^{\eta}-\omega_{j}^{\eta}\right)}\right) \mathrm{E}\left[e^{-\omega_{j}^{\eta} d_{K}^{\eta} \theta\left(\mathrm{I}+\sigma^{2}\right)}\right]
\end{aligned}
$$

where the expectation in (9) is over aggregate interference power I, i.e., over both fading and interferer locations. To evaluate the expectation $\mathrm{E}\left[e^{-d_{j}^{\eta} \theta\left(\mathrm{I}+\sigma^{2}\right)}\right]=e^{-d_{j}^{\eta} \theta \sigma^{2}} \mathrm{E}\left[e^{-d_{j}^{\eta} \theta \mathrm{I}}\right]$, we first find the Laplace transform of I, which we denote by $\mathcal{L}_{\mathrm{I}}(s)=\mathrm{E}\left[e^{-s \mathrm{I}}\right]$.

$$
\begin{aligned}
\left.\mathcal{L}_{\mathrm{I}}(s)=\mathrm{E}\left[e^{-s\left(\sum_{d_{i} \in \phi(\hat{\lambda}) \backslash B(g)} \mathbb{1}_{i} H_{i} d_{i}^{-\eta}\right.}\right)\right] \\
\stackrel{(a)}{=} \mathrm{E}\left[\prod_{d_{i} \in \phi(\hat{\lambda}) \backslash B(g)} \mathrm{E}\left[e^{-s H_{i} d_{i}^{-\eta}}\right]\right] \\
\stackrel{(b)}{=} \mathrm{E}\left[\prod_{d_{i} \in \phi(\hat{\lambda}) \backslash B(g)} \frac{1}{1+s d_{i}^{-\eta}}\right] \\
=\exp \left(-2 \pi \hat{\lambda} \int_{g}^{\infty} \frac{x}{1+s^{-1} x^{\eta}} \mathrm{d} x\right)
\end{aligned}
$$

where $B(g)$ denotes a disc of radius $g$ centered at origin, and is used to model an interference-free guard zone around the user. This is because the cluster is assumed to consist of the $K$ closest nodes and interference is due to the nodes located outside this protection zone. The inner expectation 
in $(a)$ is over fading power while the outer expectation is over the PPP $\phi$ of intensity $\lambda$ outside $B(g)$. Next, we exploit the property of independent thinning of a PPP to deal with the transmission indicator and consider a (thinned) PPP with effective density $\hat{\lambda}=p_{\mathrm{tr}, o} \lambda$. As the fading is i.i.d. across links and from further conditioning over the location, we obtain (b). The last equation follows by invoking the probability generating functional (PGFL) [16] of the PPP and some simple algebraic steps. With further algebraic manipulations, (10) can be expressed in terms of hypergeometric function, which with $s=d_{j}^{\eta} \theta$, gives

$$
\left.\mathcal{L}_{\mathrm{I}}(s)\right|_{s=d_{j}^{\eta} \theta}=\exp \left(-\pi \hat{\lambda} g^{2} \mathcal{F}\left(\frac{d_{j}^{\eta}}{g^{\eta}} \theta, \eta\right)\right)
$$

where $\mathcal{F}(\cdot, \cdot)$ is given by (7). Evaluating the expectation in (9) using (11), and further substituting $g=d_{K}, \hat{\lambda}=p_{\mathrm{tr}, o} \lambda$ and $d_{j}=\omega_{j} d_{K}$, we obtain the result in Theorem 1 .

Remark 1: Note that the above theorem allows the incluster APs to have possibly different energy harvesting rates or buffer size, and is therefore useful for getting general insights about the performance when the cluster consists of heterogeneous APs. Furthermore, all the interfering APs can be assumed to have the maximum harvesting rate in order to get a lower bound on performance. Some special cases of Theorem 1 are listed below.

- $\theta \longrightarrow 0$. In the low-outage regime, the performance is dominated by the energy harvesting parameters and the cluster size. In particular, as $\theta \rightarrow 0$ in (5), we get $\lim _{\theta \rightarrow 0} \bar{F}_{\gamma}(K, \theta)=1-Q$, where $Q$ defines a limit on the performance and infact represents the exact outage probability in the asymptotic regime. This observation also holds for Theorem 2.

- $\left\{q_{\mathrm{tr}, i}\right\}_{i=1}^{K}=q_{\mathrm{tr}, o} \equiv q_{\mathrm{tr}}$. When all the APs have similar energy harvesting capabilities, i.e., $q_{t r, i}=q_{t r, o} \equiv q_{\mathrm{tr}}$, the CCDF in (5) simplifies to

$$
\bar{F}_{\gamma}(K, \theta)=\sum_{j=1}^{K}\left(\frac{\sum_{i=0}^{K-1} \alpha_{i}(\Omega)\left(q_{\mathrm{tr}}{ }^{i}-q_{\mathrm{tr}}{ }^{K}\right)\left(\omega_{j}^{\eta}\right)^{i}}{\omega_{j}^{\eta}\left(\prod_{l \neq j}^{K} \omega_{l}^{\eta}-\omega_{j}^{\eta}\right)}\right) \Delta_{j}(\theta)
$$

where $\Delta_{j}(\theta)$ is given by (6).

Note that Theorem 1 can be used for analyzing cooperative setups in the presence of interference and noise, for a given cluster geometry. For a homogeneous network, we next provide a more general result in terms of normalized distances by unconditioning w.r.t. $d_{K}$.

Theorem 2: For a given set of normalized distances $\left\{\omega_{i}\right\}_{i=1}^{K}$, the CCDF of $\gamma, \bar{F}_{\gamma}^{\prime}(K, \theta)$, as a function of cluster size $K$ and transmission probability $p_{\text {tr }}$, in the interference- limited regime $\left(\sigma^{2} \rightarrow 0\right)$, is given by

$$
\begin{aligned}
& \sum_{j=1}^{K}\left(\frac{\sum_{i=0}^{K-1} \alpha_{i}(\Omega)\left(q_{\mathrm{tr}}{ }^{i}-q_{\mathrm{tr}}{ }^{K}\right)\left(\omega_{j}^{\eta}\right)^{i}}{\omega_{j}^{\eta}\left(\prod_{l \neq j}^{K} \omega_{l}^{\eta}-\omega_{j}^{\eta}\right)}\right)\left(1+\mathcal{F}\left(\omega_{j}^{\eta} \theta, \eta\right)\right)^{-K} .
\end{aligned}
$$

Proof: We begin the proof along the lines of [17] by leveraging a known result on PPP distance distribution. As shown in [19], the distance $d_{K}$, between a typical user and its $K^{\text {th }}$ closest AP, follows a generalized Gamma distribution, i.e.,

$$
f_{d_{K}}(r)=2(r \Gamma(K))^{-1}\left(p_{\text {tr }} \lambda \pi r^{2}\right)^{K} e^{-p_{\text {tr }} \lambda \pi r^{2}}
$$

where $\Gamma(K)$ is the Gamma function. Plugging $\sigma^{2}=0$ in (6), and taking expectation w.r.t. $d_{K}$, we get

$$
\begin{aligned}
E & {\left[\Delta_{j}(\theta)\right] } \\
& =\int_{r>0} e^{-\pi p_{\mathrm{r}} \lambda r^{2} \mathcal{F}\left(\omega_{j}^{\eta} \theta, \eta\right)} \frac{2\left(p_{\mathrm{tr}} \lambda \pi r^{2}\right)^{K} e^{-p_{\mathrm{tr}} \lambda \pi r^{2}}}{r \Gamma(K)} \mathrm{d} r \\
& =\int_{0}^{\infty} \frac{e^{-v} v^{K-1}}{\Gamma(K)\left(1+\mathcal{F}\left(\omega_{j}^{\eta} \theta, \eta\right)\right)^{K}} \mathrm{~d} v \\
& =\frac{1}{\left(1+\mathcal{F}\left(\omega_{j}^{\eta} \theta, \eta\right)\right)^{K}}
\end{aligned}
$$

where the last equation is obtained by using a dummy variable $v=r^{2}\left(1+\mathcal{F}\left(\omega_{j}^{\eta} \theta, \eta\right)\right) \pi p_{\text {tr }} \lambda$ in the integral, and then using the definition of the Gamma function $\Gamma(K)=\int_{0}^{\infty} e^{-x} x^{K-1} \mathrm{~d} x$. By taking expectation of (12) w.r.t. $d_{K}$, and using (15), we can recover the expression in Theorem 2.

Remark 2: Note that unlike Theorem 1, the CCDF expression in Theorem 2 is independent of the AP intensity $\lambda$. The probability of finding the closest AP around the receiver increases with $\lambda$, but so does the interference such that the two effects cancel out. Some special cases are postulated below.

- $p_{\text {tr }} \rightarrow 1$. It is worth noting that without energy harvesting and a random medium access protocol, i.e., as $p_{\text {tr }} \rightarrow 1$ in (13), we can retrieve the expression for the CCDF of $\gamma$ in a traditional cooperative network as given in [17], which Theorem 2 generalizes.

- $K=1$. For the non-cooperative case, the expression in (13) simplifies to $\bar{F}_{\gamma}^{\prime}(1, \theta)=\left(1-q_{\mathrm{tr}}\right)(1+\mathcal{F}(\theta, \eta))^{-1}$. Furthermore, with $q_{\mathrm{tr}}=0$, we can retrieve the CCDF expression for the signal-to-interference ratio (SIR) in a traditional non-cooperative network as given in [20].

\section{Simulation Results}

In this section, we compare the analytical model with simulation results and investigate the impact of several parameters on outage performance. 


\section{A. Validation and Comparisons}

We first consider the case with heterogeneous in-cluster APs, and plot $\bar{F}_{\gamma}(K, \theta)$, the CCDF of $\gamma$, for various values of $K$ in Fig. 2. The analytical (an) CCDF is obtained using Theorem 1, while the simulated (sim) curve is obtained by Monte Carlo simulations for the given set of parameters. The analytical model is validated since there is a complete agreement between analytical and simulation results. For comparison, the curve for the CCDF of the exact (ex) SINR obtained using Monte Carlo simulations, is also included. It can be seen that the lower bound $\gamma$ closely models the SINR distribution, particularly in the low-outage regime where most systems typically operate.

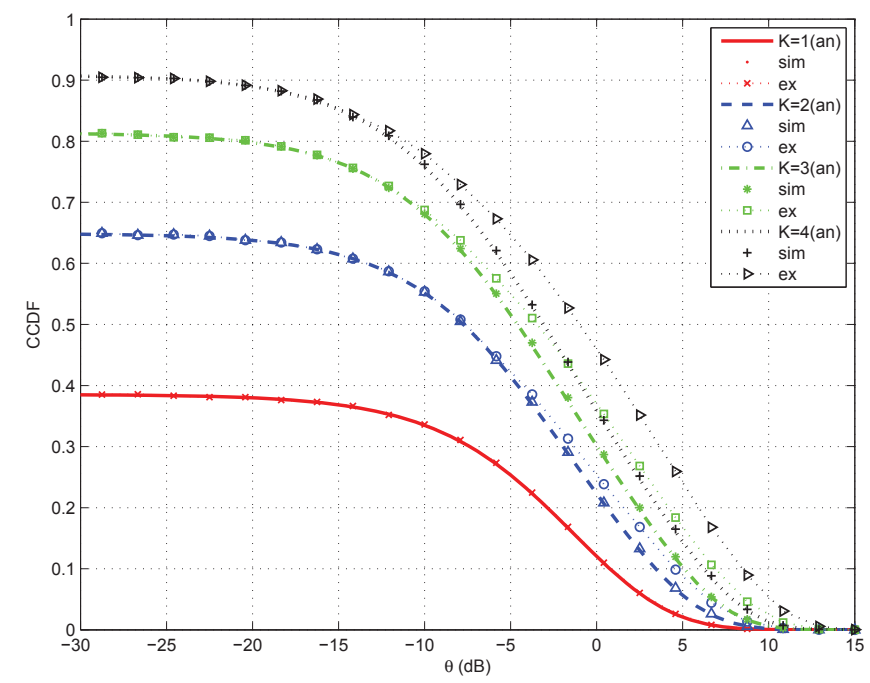

Fig. 2. $\mathrm{CCDF}$ of $\gamma$ for various values of $\mathrm{K}$ given $p_{\mathrm{ch}}=0.7, \lambda=0.1, \sigma^{2}=$ $0.01, \eta=4$ and $\left\{d_{i}\right\}_{i=1}^{4}=\{2,3,4,5\}$. Plot includes analytical (an) and simulation (sim) results for $\gamma$ as well as the simulated CCDF of exact (ex) SINR $_{u}$. Energy harvesting parameters are $\left\{\rho_{i}\right\}_{i=1}^{4}=\{0.4,0.45,0.5,0.55\}$, $\rho_{o}=0.55$ and $S=2$.

Moreover, we can also observe that the SINR distribution at the receiver improves with $K$ due to an additional transmit diversity gain. Also, the outage performance is limited by the energy harvesting capabilities as the CCDF converges to $1-Q$ in the low-outage regime $(\theta \rightarrow 0)$ for any given cluster.

Next, we consider the case where the APs have identical energy harvesting capabilities. In Fig. 3, we plot $\bar{F}_{\gamma}^{\prime}(K, \theta)$, the CCDF of $\gamma$ with the absolute in-cluster distances averaged out. It can be seen that there is a complete match between the analytical curve based on Theorem 2 and the simulated CCDF obtained via Monte Carlo simulations. Similar to Fig. 2, the CCDF of the exact SIR at the receiver is also included.

As demonstrated above, the considered framework can be used to get general performance insights for a large class of self-powered wireless networks. We next study how the energy harvesting parameters limit the outage performance.

\section{B. Impact of Energy buffer size on performance}

To get general performance insights, we use the asymptotic outage probability $Q$ as a performance metric and consider

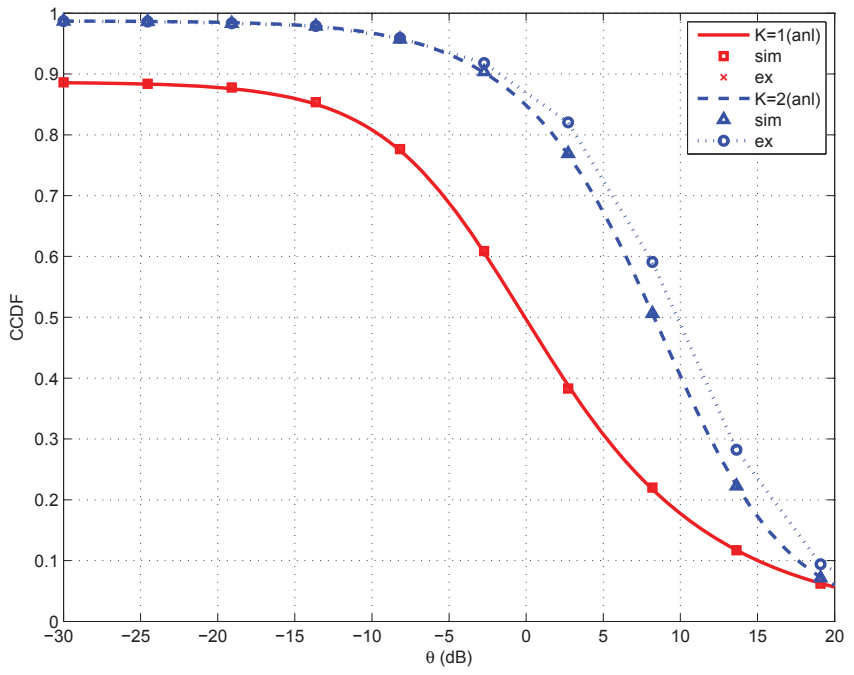

Fig. 3. CCDF of $\gamma$ in the interference-limited regime for $K \in\{1,2\}$. The plot includes the analytical (an) curve based on Theorem 2 as well as the simulated (sim) CCDF of $\gamma$. The simulated CCDF of the exact SIR at the receiver is also included. The simulation parameters are $\omega_{1}=1$ for $K=1$ and $\left\{\omega_{i}\right\}_{i=1}^{2}=\{0.5,1\}$ for $K=2, p_{\mathrm{ch}}=0.95$ and $\eta=4$. The energy harvesting parameters are $\rho=0.9$ and $S=2$ for all APs.

the case where the APs have identical energy harvesting capabilities. It should be noted that it is possible to particularize the subsequent analysis for a given outage threshold $\theta$ and/or for the case of heterogeneous APs. In Fig. 4, the asymptotic outage probability $Q$ is plotted against the energy buffer size $S$ (in log scale) for various values of the cluster size $K$. It can be seen that outage can be considerably reduced by increasing the buffer size until a limit, beyond which the curves tend to flatten out ${ }^{1}$. It appears that appreciable performance gains can be extracted with a relatively small buffer size. Moreover, the benefits of having a high-capacity buffer tends to increase with the cluster size as depicted by the increasing steepness of the slopes (when $S$ is small) as $K$ is increased. In addition, we can also observe that the outage is reduced by roughly an order of magnitude with every addition in the cluster size.

\section{Impact of Energy harvesting rate on performance}

In Fig. 5, the asymptotic outage probability $Q$ is plotted against the energy harvesting rate $\rho$ for various values of energy buffer size $S$. It can be seen that outage reduces with the increase in energy harvesting rate at the transmitters. Moreover, using a larger energy buffer brings about further reduction in outage due to enhanced energy availability at the transmitters. Furthermore, the gains from using a larger buffer size are more evident at relatively high energy harvesting rates. Fig. 5 also corroborates the previous observation (cf. Fig. 4) that substantial performance can be extracted by using a relatively small buffer size. For example, $S=10$ suffices for this setup. In addition, if the energy harvesting rate $\rho$ exceeds the channel access probability $p_{\text {ch }}$, and the buffer size

\footnotetext{
${ }^{1}$ This saturation kicks in because as $S \rightarrow \infty, p_{\mathrm{tr}}$ is limited by $\min \left\{\rho, p_{\mathrm{ch}}\right\}$, which renders $Q$ independent of $S$ in the limit $S \rightarrow \infty$.
} 


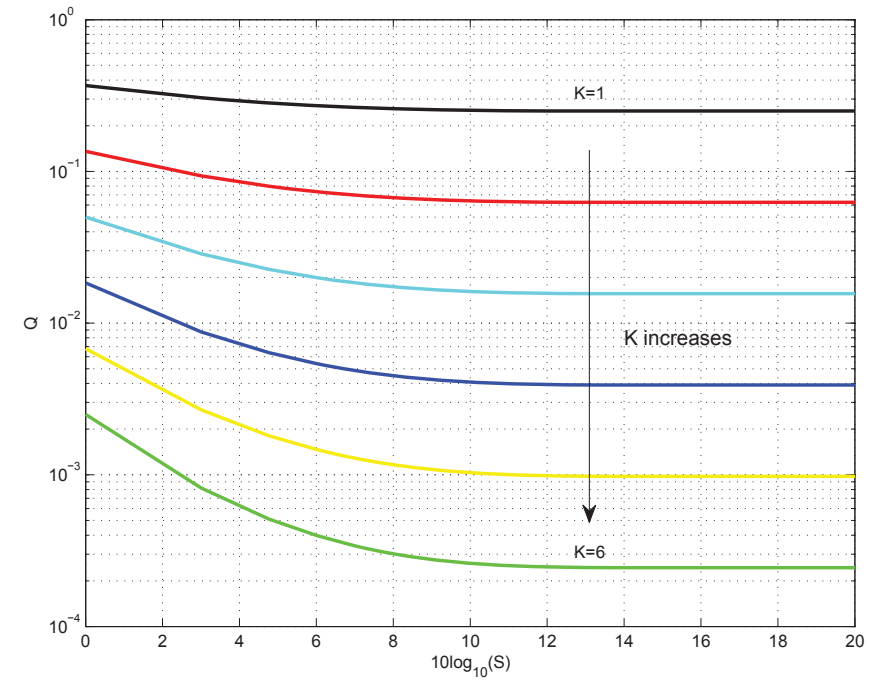

Fig. 4. Impact of energy buffer size $S$ on asymptotic outage probability $Q$ for various values of $K$ at fixed $\rho=0.75$ and $p_{\text {ch }}=0.8$.

is allowed to increase, the outage performance limit becomes independent of the energy harvesting rate $\rho$. This is because under these conditions, the energy harvesting system tends to behave like a traditionally powered system.

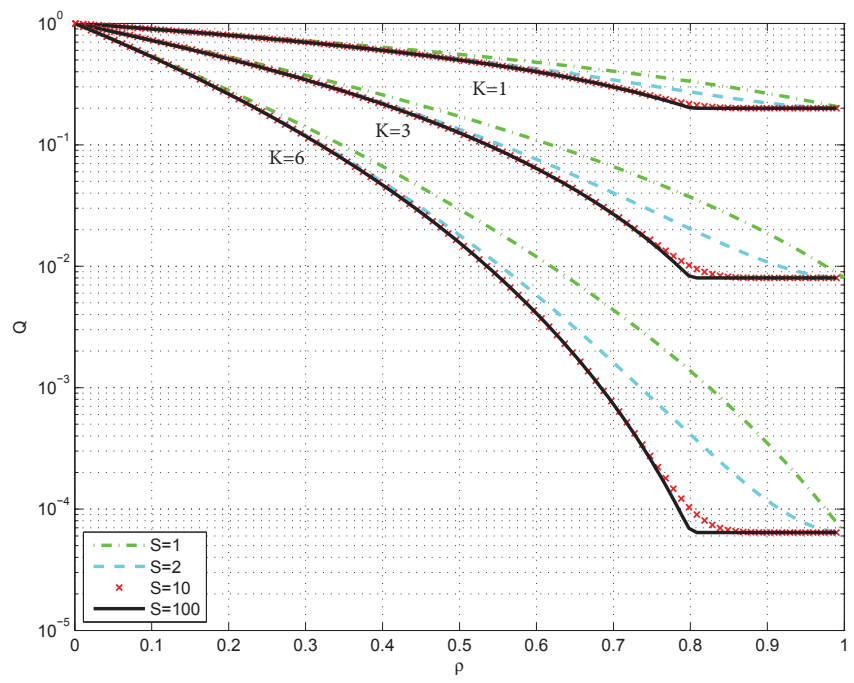

Fig. 5. Impact of energy harvesting rate $\rho$ on asymptotic outage probability $Q$ for various values of energy buffer size $S$ at fixed $p_{\text {ch }}=0.8$. The curves are plotted for cluster size $K \in\{1,3,6\}$.

\section{CONCLUSION}

We have derived closed-form expressions to characterize the outage performance at a receiver, in a self-powered clustered wireless network, in the presence of interference and noise. The developed framework is applicable to a general class of networks, with the traditional cooperative and non-cooperative networks as special cases. It can be used to get general performance insights even when the in-cluster nodes have different energy harvesting capabilities. Moreover, we have also investigated the impact of energy buffer size on performance. Simulation results reveal that the outage performance improves with the buffer size. Furthermore, most performance gains can be extracted using a relatively small buffer size, with the improvement becoming more pronounced for large clusters.

\section{REFERENCES}

[1] J. Andrews, S. Buzzi, W. Choi, S. Hanly, A. Lozano, A. Soong, and J. Zhang, "What will 5G be?," IEEE J. Sel. Areas in Commun., vol. 32, pp. 1065-1082, Jun. 2014.

[2] A. Lozano, R. Heath, and J. Andrews, "Fundamental limits of cooperation," IEEE Trans. Inf. Theory, vol. 59, pp. 5213-5226, Sep. 2013.

[3] P. Marsch and G. P. Fettweis, Coordinated Multi-Point in Mobile Communications: from theory to practice. Cambridge University Press, 2011.

[4] H. S. Rahul, S. Kumar, and D. Katabi, "JMB: Scaling wireless capacity with user demands," in Proc. ACM SIGCOMM 2012, pp. 235-246, ACM, 2012

[5] S. Venkatesan, "Coordinating base stations for greater uplink spectral efficiency in a cellular network," in IEEE Int. Symp. on Personal, Indoor and Mobile Radio Commun., pp. 1-5, IEEE, 2007.

[6] S. Sudevalayam and P. Kulkarni, "Energy harvesting sensor nodes: Survey and implications," IEEE Commun. Surveys Tuts., 2011.

[7] D. Gunduz, K. Stamatiou, N. Michelusi, and M. Zorzi, "Designing intelligent energy harvesting communication systems," IEEE Commun. Mag., vol. 52, pp. 210-216, Jan. 2014.

[8] O. Ozel, K. Tutuncuoglu, J. Yang, S. Ulukus, and A. Yener, "Transmission with energy harvesting nodes in fading wireless channels: Optimal policies," IEEE J. Sel. Areas Commun., vol. 29, pp. 1732-1743, Sep. 2011.

[9] C. K. Ho and R. Zhang, "Optimal energy allocation for wireless communications with energy harvesting constraints," IEEE Tran. Sig. Proc., vol. 60, pp. 4808-4818, Sep. 2012.

[10] K. Tutuncuoglu and A. Yener, "Sum-rate optimal power policies for energy harvesting transmitters in an interference channel," Journal of Communications and Networks, vol. 14, pp. 151-161, Apr. 2012.

[11] R. Zhang and C. K. Ho, "MIMO broadcasting for simultaneous wireless information and power transfer," IEEE Trans. Wireless Commun., vol. 12, pp. 1989-2001, May 2013.

[12] K. Huang, "Spatial throughput of mobile ad hoc networks powered by energy harvesting," IEEE Trans. Inf. Theory, vol. 59, pp. 7597-7612, Nov. 2013.

[13] R. Vaze, "Transmission capacity of wireless ad hoc networks with energy harvesting nodes," in IEEE Global Conf. on Sig. and Inf. Proc., pp. 353358, Dec. 2013

[14] S. Lee, R. Zhang, and K. Huang, "Opportunistic wireless energy harvesting in cognitive radio networks," IEEE Trans. Wireless Commun., vol. 12, pp. 4788-4799, Sep. 2013.

[15] H. Dhillon, Y. Li, P. Nuggehalli, Z. Pi, and J. Andrews, "Fundamentals of heterogeneous cellular networks with energy harvesting," IEEE Trans. Wireless Commun., vol. 13, pp. 2782-2797, May 2014.

[16] M. Haenggi, Stochastic geometry for wireless networks. Cambridge University Press, 2012.

[17] N. Lee, R. W. Heath, D. Morales-Jimenez, and A. Lozano, "Base station cooperation with dynamic clustering in super-dense cloud-RAN," in IEEE Globecom Workshops, pp. 784-788, Dec. 2013.

[18] H. Van Khuong and H.-Y. Kong, "General expression for pdf of a sum of independent exponential random variables," IEEE Commun. Lett., vol. 10, pp. 159-161, Mar. 2006.

[19] M. Haenggi, "On distances in uniformly random networks," IEEE Trans. Inf. Theory, vol. 51, pp. 3584-3586, Oct. 2005.

[20] J. Andrews, F. Baccelli, and R. Ganti, "A tractable approach to coverage and rate in cellular networks," IEEE Trans. Commun., vol. 59, pp. 31223134, Nov. 2011. 